\title{
Metodología gerencial para el mantenimiento preventivo de equipos médicos mínimos usados en habilitación de cirugías ambulatorias*
}

[Artículos de investigación]

Oryana del Pilar Villarraga Lozano**

Recibido: 12 de febrero de 2020

Revisado: o5 de agosto de 2020

Aceptado: 15 de agosto de 2020

Cómo citar este artículo:

Villarraga Lozano, O. P. (2020). Metodología gerencial para el mantenimiento preventivo de equipos médicos mínimos usados en habilitación de cirugías ambulatorias. Signos, Investigación en sistemas de gestión, 13(1). https://doi.org/10.15332/24631140.6342

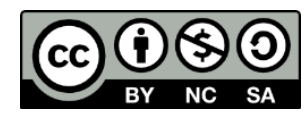

\section{Resumen}

En la gestión del mantenimiento de tecnología biomédica de los equipos médicos mínimos para la habilitación de cirugía ambulatoria, se evidencia una deficiente organización y ejecución de los mantenimientos

\footnotetext{
* Artículo de resultado de investigación

** Universidad Militar Nueva Granada. Estudiante de Maestría en Gerencia de Proyectos. Ingeniera biomédica. Universidad Militar Nueva Granada, Bogotá, Colombia. Correo electrónico: u21100132@unimilitar.edu.co, oryanavillarraga@hotmail.com. ORCID: https://orcid.org/0000-0003-0914-6308. CvLAC:

https://scienti.minciencias.gov.co/cvlac/visualizador/generarCurriculoCv.do?cod_rh $=0$ $\underline{001813874}$
} 
preventivos, lo que disminuye la disponibilidad de los equipos y aumenta las paradas y los costos en la organización de salud. El objetivo de esta metodología es orientar la gestión del mantenimiento preventivo de equipos de cirugía ambulatoria con enfoque en su disponibilidad. En la metodología propuesta, se considera el impacto operacional y global, la probabilidad de daño y el nivel de riesgo de los equipos médicos, lo que genera niveles de prioridad en la ejecución del mantenimiento preventivo. Según los resultados obtenidos, se identifica que, para cirugías ambulatorias, los tres equipos que presentan un nivel de riesgo alto asociado a su disponibilidad son la máquina de anestesia, el electrobisturí y la lámpara cielítica. Su mantenimiento debe realizarse de manera prioritaria, ya que el impacto de su carencia será alto. Se concluye que es necesario contar con equipos de respaldo - sobre todo para los que presentan mayor nivel de riesgo-, tener información verídica de la existencia de los equipos, estandarizar procesos, crear procedimientos y protocolos, y capacitar al personal encargado de la tecnología.

Palabras clave: cirugía ambulatoria, mantenimiento preventivo, disponibilidad del equipo, metodología gerencial.

\section{Management methodology for the preventive maintenance of minimum medical equipment used in the qualification of ambulatory surgeries}

\section{Abstract}

In the maintenance of biomedical technology of the minimum medical equipment for the qualification of ambulatory surgery, a deficient organization and execution of the preventive maintenance is evidenced, which diminishes equipment availability and increases stops and costs in the health provider organization. This methodology seeks guiding the management of preventive maintenance of ambulatory surgery equipment focused on the equipment availability. The proposed methodology takes into account the operational and global impact, the 
damage probability, and the level of risk of the medical equipment, thus generating priority levels in the preventive maintenance execution. Based on the results obtained, it is identified that, for ambulatory surgeries, the three equipment with a high level of risk associated to their availability are the anesthesia machine, the electro-scalpel, and the surgery lamp. This equipment maintenance must be a priority since the impact of their unavailability will be high. It is concluded that it is necessary to have backup equipment-especially for those with a higher level of risk-to have accurate information about the equipment existence, to standardize processes, to create procedures and to train the personnel in charge of technology.

Keywords: ambulatory surgery, preventive maintenance, equipment availability, management methodology

\section{Metodologia de gestão para a manutenção preventiva de equipamentos médicos mínimos utilizados na qualificação de cirurgias ambulatoriais}

\section{Resumo}

Na manutenção da tecnologia biomédica do equipamento médico mínimo para a qualificação da cirurgia ambulatorial, evidencia-se uma organização e execução deficientes da manutenção preventiva, reduzindo a disponibilidade do equipamento e aumentando as paradas e os custos na organização da saúde. O objetivo desta metodologia é nortear a gestão da manutenção preventiva de equipamentos de cirurgia ambulatorial com foco em sua disponibilidade. Na metodologia proposta, são considerados o impacto operacional e geral, a probabilidade de prejuízo e o nível de risco do equipamento médico, o que gera níveis prioritários na execução da manutenção preventiva. De acordo com os resultados obtidos, identifica-se que, para cirurgias ambulatoriais, os três equipamentos que apresentam um alto nível de risco associado à sua disponibilidade são o equipamento de anestesia, o eletrobisturi e a 
lâmpada cialítica. A sua manutenção deve ser feita prioritariamente, pois o impacto de sua falta será alto. A conclusão é que é necessário ter equipamentos de apoio, especialmente para aqueles com maior nível de risco, ter informações precisas sobre a existência dos equipamentos, padronizar os processos, criar procedimentos e treinar o pessoal encarregado dessa tecnologia.

Palavras-chave: cirurgia ambulatorial, manutenção preventiva, disponibilidade do equipamento, metodologia de gestão.

\section{Introducción}

La gestión del mantenimiento ha tenido gran impacto sobre el desarrollo de las entidades prestadoras de salud y la calidad en la atención a los usuarios, y con ella, han surgido temas regulatorios y legales que requieren obligatorio cumplimiento. No obstante, las dificultades en el área de la salud no han sido subsanadas totalmente a pesar de los controles ejecutados; por esta razón, surgen inconformidades que afectan silenciosamente al sector salud del país.

Es válido resaltar que actualmente no existe una metodología gerencial para la gestión de mantenimiento preventivo y cada institución lo realiza de la manera más adecuada según sus propios criterios. Sin embargo, el seguimiento y la aplicación de esta gestión no genera resultados satisfactorios porque aún se evidencian fallas frecuentes en los equipos, se presentan tiempos de parada altos y no se ejecuta una renovación tecnológica apropiada según la vida útil que dicta el fabricante de los equipos.

Para obtener una buena atención es importante, además de nombrar al personal asistencial, contar con equipos médicos funcionales que se encuentren disponibles en las instituciones prestadoras de salud (ips). Por esta razón, la gestión del departamento de ingeniería biomédica en el 
mantenimiento diseñado y aplicado de los equipos médicos requiere efectividad. Es necesario proponer una metodología gerencial para el mantenimiento preventivo de los equipos médicos mínimos en habilitación de cirugía ambulatoria, que priorice la disponibilidad de estos equipos y asegure, así, las condiciones mínimas de funcionamiento para una ips.

Cuando se habla de activos físicos, es fundamental establecer una verificación que conlleve la observación, la predicción, las recomendaciones de fabricante, la legislación y la seguridad del paciente, ya que de esta manera se puede definir una periodicidad de mantenimiento preventivo para estos elementos y lograr una reducción de fallas y sus derivaciones (Sobral y Soares, 2016). Así, la gestión de tecnología biomédica (Castaño, 2015) es de gran importancia y ha tenido su reconocimiento en diferentes escenarios como un elemento más del sistema de salud actual.

Es necesario definir criterios para priorizar los equipos médicos presentes en una ips. Estos criterios pueden ser: función del equipo (sostenimiento de vida, tratamiento, monitoreo, etc.), aplicación clínica (incluyendo riesgo del equipo), requerimientos de mantenimiento e historial de fallas (por cada correctivo) (Rodríguez et ál., 2001). A cada criterio definido se le asigna un valor, estos valores se suman y su resultado clasifica la criticidad e importancia del equipo. Otra opción para priorizar consiste en definir parámetros de severidad, ocurrencia y detección del mantenimiento preventivo, su planificación, diseño, actividades y análisis de riesgo (Lemos et ál., 2012). Se asigna un valor numérico, teniendo en cuenta el equipo que se está evaluando.

Las ips requieren contar con información válida y verídica sobre el estado de los equipos médicos utilizados en la atención a los usuarios. Como no hay metodología gerencial definida en este sector, se hace necesario 
diseñarla y aplicarla, teniendo en cuenta estándares del Project Management Body of Knowledge (pmbok) e iso 21500. De esta manera, se podrá mejorar la oportunidad en la atención en salud, aumentar la disponibilidad total de los equipos para los pacientes, reducir las fallas y tomar decisiones de mantenimiento considerando tanto la disponibilidad del equipo como su riesgo.

\section{Metodología}

Para la ejecución de la metodología gerencial que prioriza la disponibilidad de los equipos, se manejó un tipo de estudio exploratorio y diseño documental. Se tuvieron en cuenta cinco áreas de conocimiento que fueron seleccionadas según la importancia del mantenimiento, la disponibilidad de equipos y la comparación de los estándares del pmbok e iso 21500. Las cinco áreas de conocimiento seleccionadas fueron: tiempo, costo, calidad, comunicaciones y recursos. A partir de estas áreas, se diseñó el modelo teniendo en cuenta impactos, probabilidades y como base la Guía para realizar el análisis de impacto de negocios BIA del Ministerio de Tecnologías de la Información y las Comunicaciones (2015).

\section{Metodología gerencial para la disponibilidad de los equipos médicos}

Este modelo se aplicó a cada unidad de cirugía ambulatoria de las instituciones participantes. Cada institución implementó el modelo, independientemente de si pertenecía a la misma entidad promotora de salud o no.

Identificación de equipos utilizados en la unidad de cirugía ambulatoria

Relación de los equipos que se encuentran en la unidad de cirugía ambulatoria de la institución de salud. 
Evaluación de impactos operacionales (equipo vs. cirugía)

El objetivo de este paso consiste en determinar el impacto de cada uno de los equipos disponibles en la unidad de cirugía ambulatoria y evaluar qué tan necesario es el equipo médico. Para cada una de las cirugías que se realizan en las instituciones evaluadas y que se listaron en el paso anterior, se diligencia la matriz de impactos operacionales (tabla 1). Se establecieron tres niveles de impacto así:

Nivel A. Si no se tiene el equipo, la cirugía no puede realizarse.

Nivel B. Es aconsejable contar con el equipo para la cirugía, pero se puede realizar si no se tiene.

Nivel C. No se necesita el equipo para la cirugía.

La matriz se diligencia con la información reportada por el equipo médico y auxiliar de la unidad de cirugía ambulatoria. Para cada pareja equipocirugía se asigna una de las opciones A, B o C.

Tabla 1. Matriz de impactos operacionales

\begin{tabular}{|l|l|l|l|l|l|} 
& $\begin{array}{c}\text { Cirugía 1 } \\
(\mathbf{A}, \mathbf{B}, \mathbf{C})\end{array}$ & $\begin{array}{c}\text { Cirugía 2 } \\
(\mathbf{A}, \mathbf{B}, \mathbf{C})\end{array}$ & $\begin{array}{c}\text { Cirugía 3 } \\
(\mathbf{A}, \mathbf{B}, \mathbf{C})\end{array}$ & $\ldots$ & \multicolumn{1}{c|}{$\begin{array}{c}\text { Cirugía 10 } \\
(\mathbf{A}, \mathbf{B}, \mathbf{C})\end{array}$} \\
\hline Equipo 1 & $\ldots$ & $\ldots$ & $\ldots$ & $\ldots$ & $\ldots$ \\
\hline Equipo 2 & $\ldots$ & $\ldots$ & $\ldots$ & $\ldots$ & $\ldots$ \\
\hline$\ldots$ & $\ldots$ & $\ldots$ & $\ldots$ & $\ldots$ & $\ldots$ \\
\hline$\ldots$ & $\ldots$ & $\ldots$ & $\ldots$ & $\ldots$ & $\ldots$ \\
\hline Equipo 10 & $\ldots$ & $\ldots$ & $\ldots$ & $\ldots$ & $\ldots$ \\
\hline
\end{tabular}

Fuente: elaboración propia.

Evaluación de impacto global por equipo

A partir de la información de la matriz de impactos operacionales, se diligencia la matriz de impacto global (tabla 2), que consiste en resumir la importancia del equipo para las cirugías que se realizan en la unidad de 
cirugía ambulatoria. Teniendo en cuenta que no todos los equipos son indispensables en todas las cirugías ambulatorias, se definen tres niveles de prioridad de mantenimiento; el nivel alto (5) corresponde a los equipos que sean más indispensables en la unidad de cirugía ambulatoria y el nivel bajo (1) corresponde a los equipos con menor impacto operacional:

- $\quad$ Alto (5): el equipo tiene al menos en la mitad de las cirugías impacto operacional tipo A, de acuerdo con la matriz de impactos operacionales.

- $\quad$ Medio (3): el equipo tiene al menos en la cuarta parte de las cirugías impacto operacional tipo A, pero no alcanza al nivel alto, de acuerdo con la matriz de impactos operacionales.

- $\quad$ Bajo (1): el equipo tiene en menos de la cuarta parte de las cirugías impacto operacional $\mathrm{A}$, de acuerdo con la matriz de impactos operacionales.

Tabla 2. Matriz de impacto global

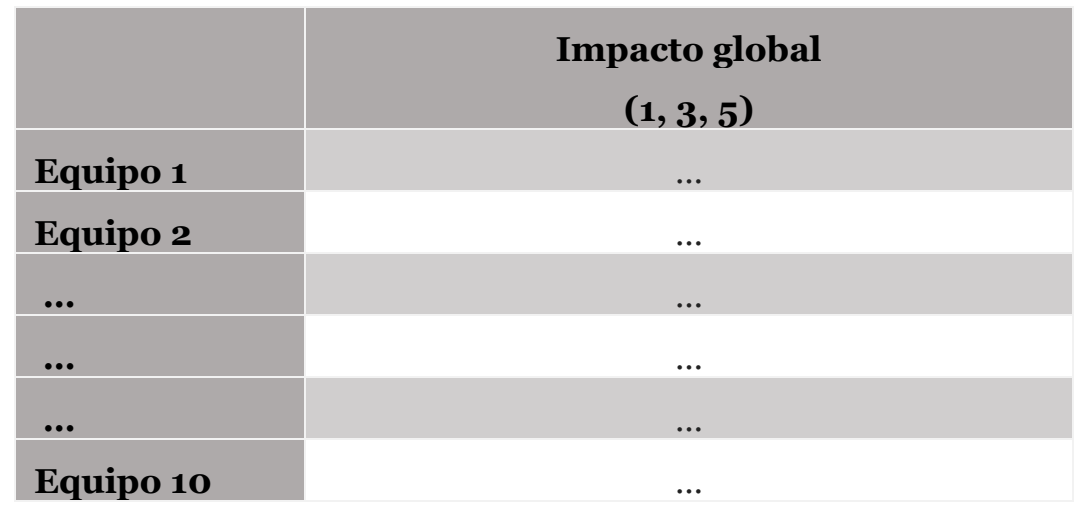

Fuente: elaboración propia.

Evaluación de la probabilidad de daño por equipo

Además de la importancia del equipo médico dentro de una unidad de cirugía ambulatoria, es necesario contemplar la periodicidad de daño del equipo si no se realiza el mantenimiento preventivo. Se definen cinco 
niveles de probabilidad de daño, de acuerdo con lo reportado por los técnicos e ingenieros del área biomédica entrevistados. Si un equipo se daña con mucha frecuencia y si no se realiza el mantenimiento preventivo, deberá tener mayor prioridad de mantenimiento para garantizar su disponibilidad que un equipo con menor probabilidad de daño en las mismas condiciones. De esta forma, el mayor valor asignado corresponde al equipo con probabilidad de daño muy frecuente y el menor valor, al caso improbable:

- Improbable (1): se daña el equipo cada dos años si no se realiza mantenimiento preventivo.

- Ocasional (2): se daña el equipo cada año si no se realiza mantenimiento preventivo.

- $\quad$ Moderado (3): se daña el equipo cada nueve meses si no se realiza mantenimiento preventivo.

- $\quad$ Frecuente (4): se daña el equipo cada seis meses si no se realiza mantenimiento preventivo.

- $\quad$ Muy frecuente (5): se daña el equipo cada tres meses si no se realiza mantenimiento preventivo.

Con la información que reporte el personal técnico de ingeniería biomédica de la institución, se diligencia la tabla 3, correspondiente a la probabilidad de daño.

Tabla 3. Probabilidad de daño del equipo sin mantenimiento preventivo

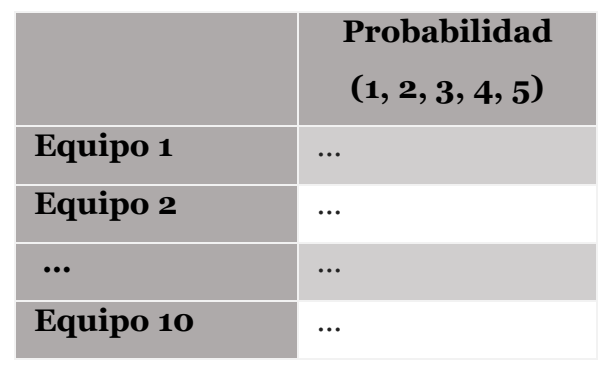

Fuente: elaboración propia. 
Es importante tener en cuenta las recomendaciones que establece el fabricante de cada equipo médico sobre la periodicidad de los mantenimientos preventivos.

\section{Evaluación del nivel de riesgo asociado a la disponibilidad de equipos}

El nivel de riesgo asociado a la disponibilidad contempla de forma simultánea el impacto global del equipo y la probabilidad de daño. Los equipos con mayor impacto y probabilidad de daño deberán tener mayor prioridad. Esto significa que si es muy probable que un equipo se dañe si no se realiza el mantenimiento preventivo y este es indispensable en la unidad de cirugía ambulatoria, tendrá el primer lugar para que se le realice mantenimiento para garantizar su disponibilidad.

Teniendo en cuenta el impacto global (tabla 2) y la probabilidad de daño (tabla 3), se diligencia la tabla 4, que corresponde al riesgo asociado a la disponibilidad, multiplicando los dos valores anteriores por cada equipo.

Tabla 4. Matriz de riesgo asociado a la disponibilidad

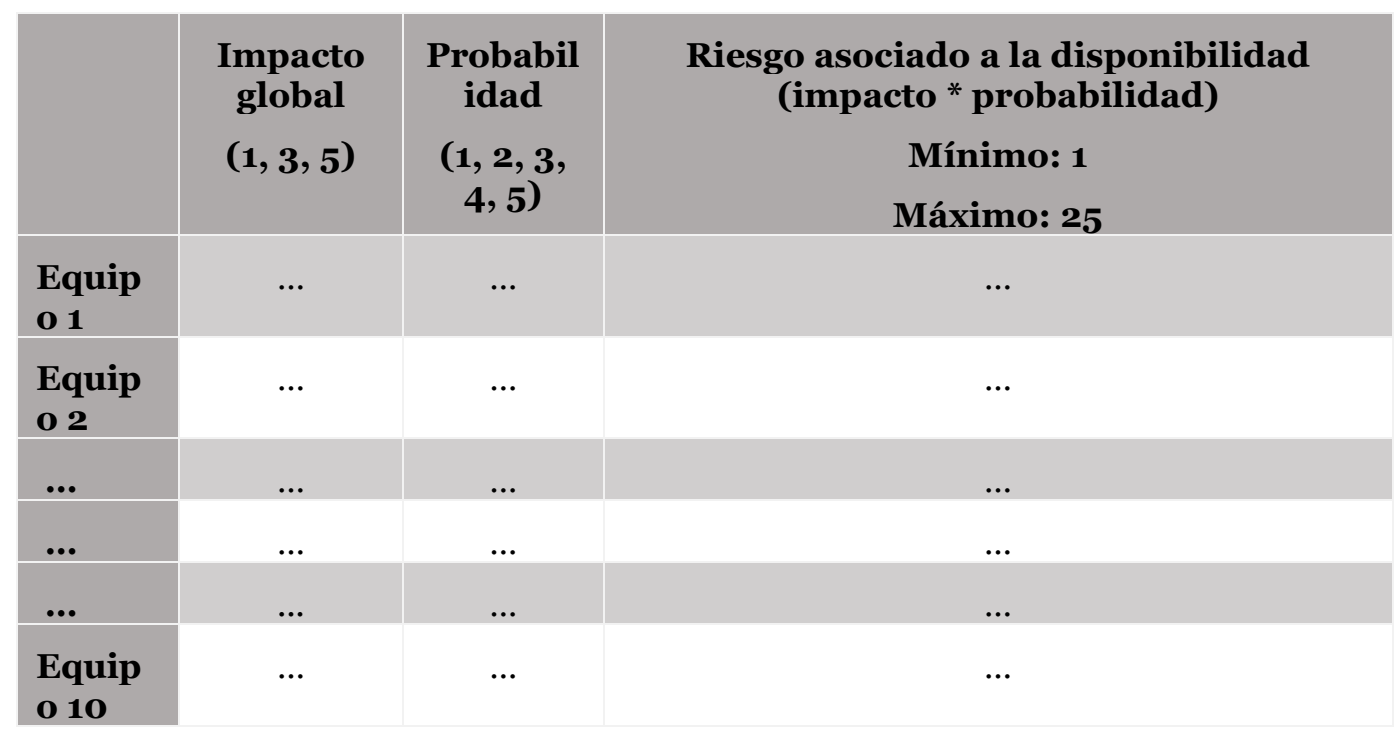

Fuente: elaboración propia. 


\section{Informe de prioridad de mantenimiento}

En este informe se ordenan los equipos en términos del riesgo asociado a la disponibilidad, teniendo en cuenta la importancia del equipo dentro de la unidad de cirugía ambulatoria y la frecuencia de daño. Se sugiere que los equipos que se encuentren en los primeros lugares del informe de prioridad de mantenimiento cuenten con un equipo de respaldo, que puede tener características menos especializadas, para cubrir las necesidades del servicio mientras el equipo no se encuentre disponible. La figura 1 resume la metodología propuesta.

Figura 1. Metodología para el mantenimiento preventivo en unidad cirugía ambulatoria que prioriza la disponibilidad de equipos

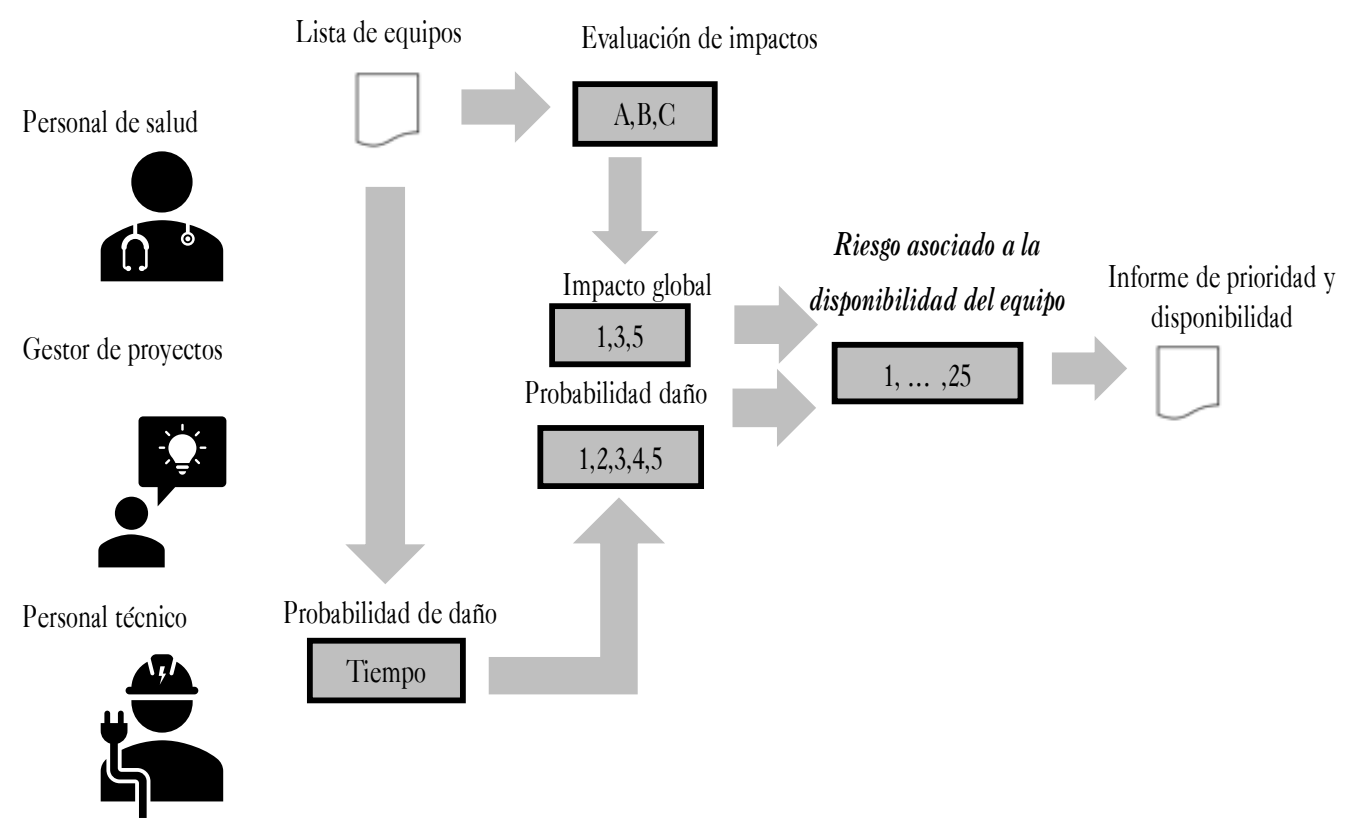

Fuente: elaboración propia.

\section{Resultados y discusión}

Se implementó y se validó la metodología gerencial diseñada con el personal experto en el área, tanto de salud como de gestión de mantenimiento, por medio de entrevistas a 10 expertos de cada área que 
pertenecían a diferentes instituciones de salud con cirugías ambulatorias habilitadas. Los equipos requeridos en una sala ambulatoria según los puntajes más altos arrojados por las entrevistas fueron: máquina de anestesia, mesa de cirugía, electrobisturí y lámpara cielítica (figura 2).

Figura 2. Equipos requeridos en sala ambulatoria

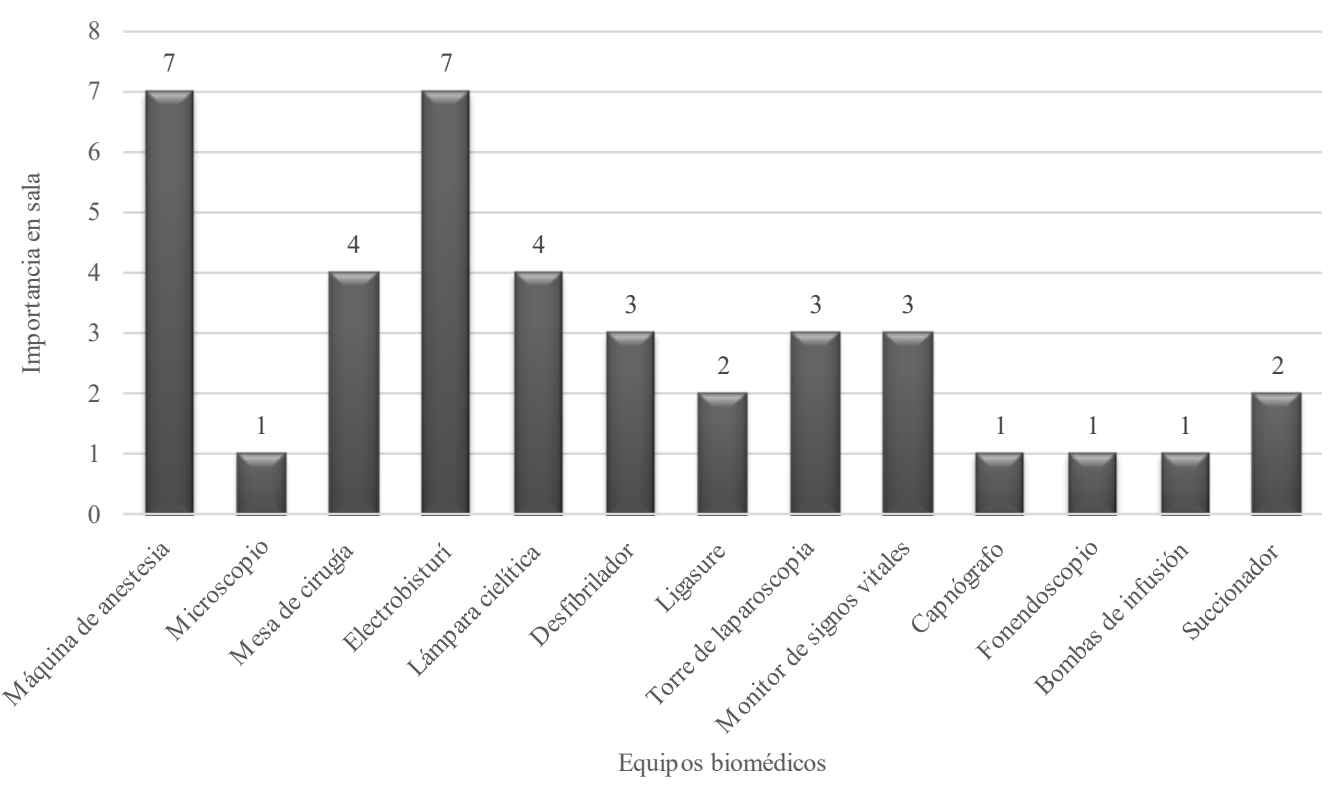

Fuente: elaboración propia.

De igual manera, se enumeraron las cirugías más frecuentes en los quirófanos al momento de implementar la metodología. Se evidenció que, según el personal experto, las cirugías más comunes fueron: hernia umbilical e inguinal, etmoidectomías, resección de masas malignas, colecistectomía, reducción de fracturas y timpanoplastias (figura 3). 
Figura 3. Cirugías realizadas en salas ambulatorias

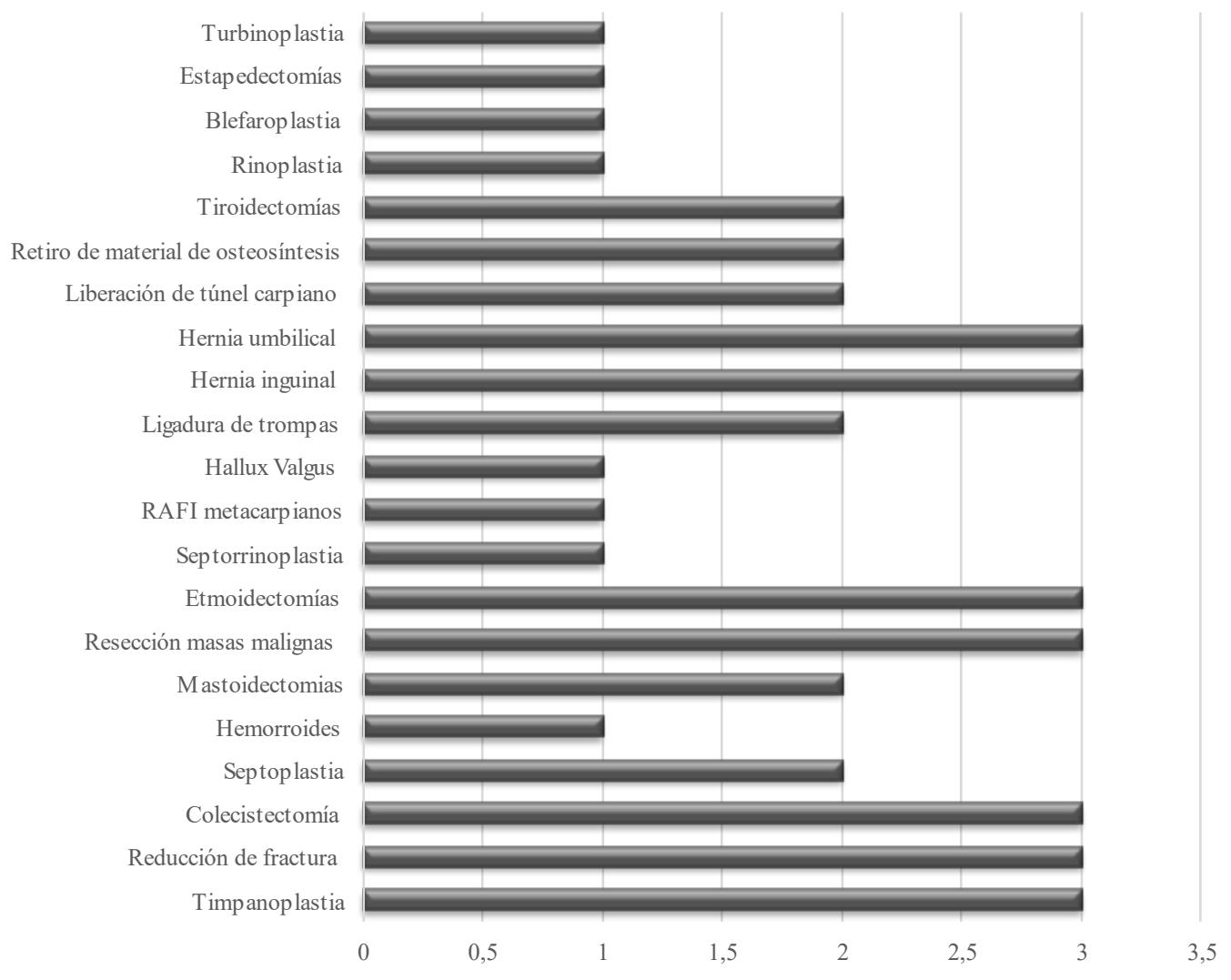

Fuente: elaboración propia.

Posteriormente, al tener definidos los equipos médicos y las cirugías más

frecuentes en una sala ambulatoria fue posible identificar el impacto

operacional de cada equipo disponible en la unidad según la clasificación

ya establecida (tabla 5 ).

Tabla 5. Impacto operacional de cirugías ambulatorias vs. equipos médicos

\begin{tabular}{|l|c|c|c|c|c|}
\hline \multicolumn{1}{|c|}{ Equipo } & $\begin{array}{c}\text { Hernia } \\
\text { inguinal }\end{array}$ & $\begin{array}{c}\text { Reducción de } \\
\text { fracturas }\end{array}$ & $\begin{array}{c}\text { Colecistect } \\
\text { omía }\end{array}$ & $\begin{array}{c}\text { Timpanopl } \\
\text { astias }\end{array}$ & $\begin{array}{c}\text { Etmoidecto } \\
\text { mías }\end{array}$ \\
\hline $\begin{array}{l}\text { Máquina de } \\
\text { anestesia }\end{array}$ & A & B & A & A & B \\
\hline Electrobisturí & A & B & A & B & B \\
\hline
\end{tabular}

SIGNOS, Investigación en Sistemas de Gestión

ISSN: 2145-1389 | e-ISSN: 2463-1140 | DOI: https://doi.org/10.15332/24631140

Vol. 13 N.o 1 | enero-junio de 2021 


\begin{tabular}{|l|c|c|c|c|c|}
\hline \multicolumn{1}{|c|}{ Equipo } & $\begin{array}{c}\text { Hernia } \\
\text { inguinal }\end{array}$ & $\begin{array}{c}\text { Reducción de } \\
\text { fracturas }\end{array}$ & $\begin{array}{c}\text { Colecistect } \\
\text { omía }\end{array}$ & $\begin{array}{c}\text { Timpanopl } \\
\text { astias }\end{array}$ & $\begin{array}{c}\text { Etmoidecto } \\
\text { mías }\end{array}$ \\
\hline Lámpara cielítica & A & B & A & C & B \\
\hline Mesa de cirugía & A & B & A & A & B \\
\hline $\begin{array}{l}\text { Monitor } \\
\text { multiparámetros }\end{array}$ & A & B & A & A & B \\
\hline $\begin{array}{l}\text { Torre de } \\
\text { laparoscopia }\end{array}$ & B & B & A & $\begin{array}{c}\text { No } \\
\text { seleccionada }\end{array}$ & $\begin{array}{c}\text { No } \\
\text { seleccionada }\end{array}$ \\
\hline
\end{tabular}

Fuente: elaboración propia.

La tabla 5 muestra que los equipos médicos relacionados con las cirugías ambulatorias tuvieron un gran impacto. Las cirugías más afectadas si no cuentan con la totalidad de los equipos en buen funcionamiento son la cirugía de hernia inguinal y la colecistectomía. No obstante, esto no asegura que para las otras cirugías no sean importantes estos equipos, solo que es posible que puedan sustituirse o utilizar otra técnica para ejecutar el procedimiento quirúrgico.

El impacto global obtenido de los equipos con puntajes más altos requeridos en una sala ambulatoria se muestra en la figura 4 .

Figura 4. Impacto global de los equipos médicos

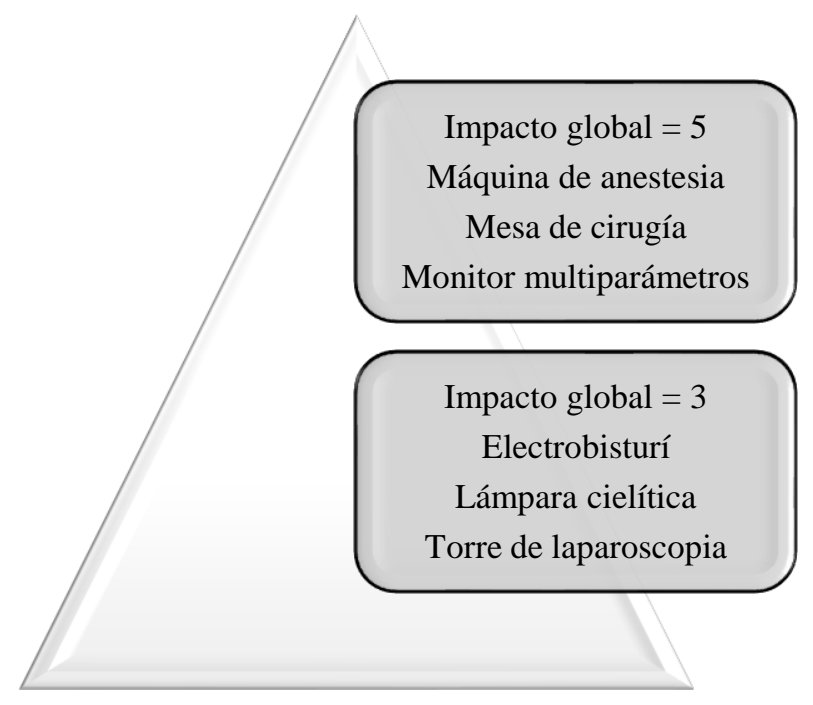

Fuente: elaboración propia. 
El impacto más alto según las entrevistas se tuvo para la máquina de anestesia, la mesa de cirugía y el monitor multiparámetros, ya que si no tienen estos equipos las cirugías no pueden ejecutarse. Los equipos con impacto global de nivel 3 podrían ser reemplazados en algunas ocasiones.

La periodicidad del mantenimiento preventivo para los equipos con los puntajes más altos de repetitividad en las entrevistas se muestra en la figura 5 .

Figura 5. Periodicidad de mantenimiento preventivo
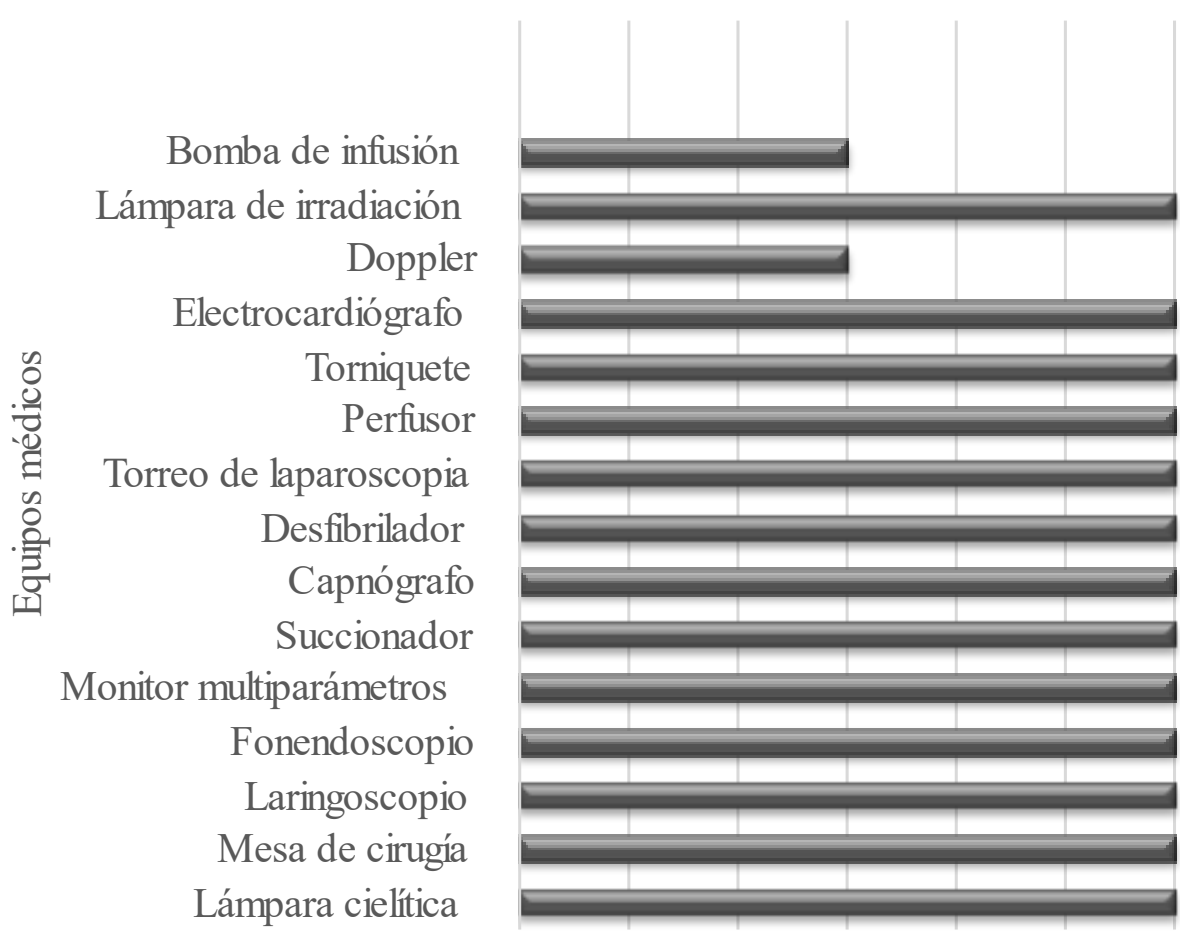

0

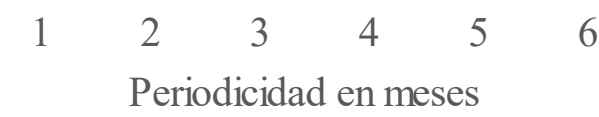

Fuente: elaboración propia.

Según la figura 5, se evidenció que la periodicidad del mantenimiento preventivo del $86 \%$ de los equipos evaluados se encuentra en seis meses. Los expertos sustentaron que es importante tener en cuenta la vida útil del 
equipo, el costo de mantenimiento, las recomendaciones del fabricante, la legislación vigente, la seguridad del paciente y la importancia de este en los quirófanos ambulatorios. Al relacionar estas periodicidades, fue posible generar una relación entre la probabilidad de riesgo vs. el impacto (tabla 6).

Tabla 6. Impacto, probabilidad y riesgo de los equipos médicos

\begin{tabular}{|l|c|c|c|}
\hline & $\begin{array}{c}\text { Impacto } \\
\text { global }\end{array}$ & $\begin{array}{c}\text { Probabilid } \\
\text { ad }\end{array}$ & $\begin{array}{c}\text { Riesgo asociado a la } \\
\text { disponibilidad }\end{array}$ \\
\hline Máquina de anestesia & 5 & 5 & 25 \\
\hline Electrobisturí & 3 & 4 & 12 \\
\hline Lámpara cielítica & 3 & 4 & 12 \\
\hline Mesa de cirugía & 5 & 4 & 20 \\
\hline $\begin{array}{l}\text { Monitor } \\
\text { multiparámetros }\end{array}$ & 5 & 4 & 20 \\
\hline Torre de laparoscopia & 3 & 4 & 12 \\
\hline
\end{tabular}

Fuente: elaboración propia.

La máquina de anestesia sigue ocupando un lugar muy importante si no recibe una óptima gestión en el mantenimiento preventivo, seguida de la mesa de cirugía y el monitor multiparámetros. De esta forma, se observó que se debe ejecutar el mantegnimiento preventivo a los equipos que tienen una mayor probabilidad de dañarse y un mayor impacto si no están disponibles en los quirófanos ambulatorios.

Con los anteriores resultados, fue posible entregar un informe acerca de la ejecución del mantenimiento preventivo de los equipos, teniendo en cuenta la disponibilidad.

\section{Conclusiones}

La metodología gerencial que prioriza la disponibilidad de los equipos médicos mostró un panorama enfocado en la operatividad, considerando probabilidades de afectación del servicio según las fallas presentadas si no 
se realiza una buena gestión en el mantenimiento preventivo. La Guía para realizar el análisis de impacto de negocios BIA del MinTIC que se utilizó como base para el desarrollo de la metodología permitió tener más control en los parámetros y generar impactos y posibles riesgos relacionados con el mantenimiento ejecutado en los equipos.

Se estableció que para los equipos que presentaron una importancia alta en las salas de cirugía ambulatoria, es necesario contar con un equipo de back-up. Estos dispositivos pueden poseer características menores, pero logran suplir la necesidad en el momento de que se presente algún percance en los quirófanos ambulatorios.

Con respecto a la gestión del mantenimiento, es necesario estandarizar los procesos, crear y proponer manuales, procedimientos y protocolos que permitan guiar al personal para aprovechar todos los recursos presentes, tecnología biomédica y herramientas de trabajo. Los manuales se deben complementar con capacitaciones para el personal encargado de los equipos. Según los expertos entrevistados, en la validación del entregable se podría tener en cuenta la cantidad de equipos de back-up disponibles en la institución, criterios de fábrica y chequeos periódicos a las áreas o servicios en cuestión.

\section{Referencias}

Castaño C. (2015). Análisis comparativo de modelos de gestión de tecnología biomédica. Revista Ingeniería Biomédica, 9(18), 41-49.

Lemos, L., Brasil, L., Silva, J. y Elpídio, F. (2012). Biomedical devices project implementation with predictive management. 2012 Pan American Health Care Exchanges (PAHCE). Conference, Workshops, and Exhibits.

Ministerio de Tecnologías de la Información y las Comunicaciones. (2015). Guía para realizar el análisis de impacto de negocios BIA. Guía N. ${ }^{\circ} 11$. 
Organización Internacional para la Normalización. (2012). ISO 2150o. Orientación sobre la gestión de proyectos.

Rodríguez, E., Miguel, A. y Sánchez, M. (2001). Gestión de mantenimiento para equipos médicos. II Congreso Latinoamericano de Ingeniería Biomédica, La Habana, Cuba. https://pdfs.semanticscholar.org/a3e3/5ed9870dbb92d18a1ado3a6833e3f4d4828 b.pdf

Sobral, J. y Soares, C. (2016). Preventive maintenance of critical assets based on degradation mechanisms and failure forecast. IFAC Papers Online, 49(28), 97-102.

\section{Bibliografía consultada}

Alban, L. y Lara, E. (2017). Propuesta de metodología para gestión de mantenimiento de equipos y sistema de uso médico. Revista Publicando, 1O(2), 143-153.

Ardila, J., Ardila, M., Rodríguez, D. y Hincapié, D. (2016). Gerencia del mantenimiento: una revisión. Dimensión Empresarial, 14(2), 127-142. http://dx.doi.org/10.15665/rde.v14i2.480

Avendaño, M. (2010). Elaboración de un programa de capacitación para el uso y rutina diaria de los equipos por los usuarios de acuerdo a los requisitos de la Joint Comission International. Instituto Politécnico Nacional.

Chompu-Inwai, R., Tipgunta, S. y Sunawan., A. (2008). Implementation of total productive maintenance in healthcare: a pilot study. International Conference on Service Systems and Service Management, Melbourne, Australia.

Congreso de la República. (1993, 23 de diciembre). Ley 100 de 1993. Por el cual se crea el Sistema de seguridad social integral y se dictan tras disposiciones. Diario Oficial 41148. https://docs.supersalud.gov.co/PortalWeb/Juridica/Leyes/Lo10o_93.pdf

Congreso de la República. (2011, 19 de enero). Ley 1438 de 2011. Por medio del cual se reforma el Sistema General de Seguridad Social en Salud y se dictan otras disposiciones. Diario Oficial 47957. https://www.minsalud.gov.co/Normatividad Nuevo/LEY\%201438\%20DE\%2020 11.pdf 
Congreso de la República. (2015, 16 de febrero). Ley 1751 de 2015. Por medio Del cual se regula el derecho fundamental a la salud y se dictan otras disposiciones. Diario Oficial 49427.

https://www.minsalud.gov.co/Normatividad_Nuevo/Ley\%201751\%20de\%202015. pdf

Congreso de la República. (1979, 16 de julio). Ley 9 de 1979. Por medio del cual se dictan medidas sanitarias. Diario Oficial 35308.

https://www.minsalud.gov.co/Normatividad Nuevo/LEY\%200009\%20DE\%2019 79.pdf

Coulibaly, S., Kouam, P. y Page M. (2001). Organisation de la maintenance préventive en milieu hospitalier. ITBM-RBM, 22(2), 125-133.

Duarte, F. y Diaz, J. (2015). Plan de gestión de proyectos de mantenimientos mayores de equipos en las estaciones de bombeo de ecopetrol S. A. [Tesis de especialización]. Universidad Industrial de Santander.

Escola d'Oficis Catalunya. (2018). Tipos de cirugía y su clasificación. https://escoladoficiscatalunya.com/tipos-de-cirugias-clasificacion/

García, W., Orozco, W., Narváez, J. y Palacio, J. (2017). Gerencia de mantenimiento, metrología y producción más limpia en equipos y dispositivos médicos en instituciones de salud de Medellín, Colombia. Revista Espacios, 38(52), 28.

Gismondi, G. (2010). Ingeniería biomédica. Revista Ciencia y Cultura, 24, 99-118.

Han, Y., Zhen, X., Huan, Y. y Vinnem, J. (2019). Integrated methodology for determination of preventive maintenance interval of safety barriers on offshore installations. Process Safety and Environmental Protection, 132, 313-324. https://doi.org/10.1016/j.psep.2019.09.035

Hepp, J., Csendes, A., Ibañez, F., Llanos, O. y San Martín, S. (2008). Programa de la especialidad cirugía general. Definiciones y propuestas de la sociedad de cirujanos de Chile. Revista Chilena de Cirugía, 6o(1), 79-85.

Hernández, H., Fernández C. y Baptista, P. (2014). Metodología de la investigación. McGrawHill Education. 
Hincapié, L. (2017). Metodología de gestión de mantenimiento desde una perspectiva de confiabilidad-disponibilidad-mantenibilidad (CDM) para la aplicación de equipos de tecnología de la información (TI) [Tesis de maestría]. Universidad Nacional de Colombia.

Hurtado, J. (2014). Cómo formular objetivos de investigación: un acercamiento desde la investigación holística. Editorial Neisa.

Hyllarick. (2012). Ingeniería biomédica. Prótesis primitivas. http://hbiomedica.blogspot.com/p/protesis-primitivas.html

International Project Management Association. (2015). Individual competence baseline for Project Management. IPMA.

Invima y Ministerio de Salud y Protección Social. (2012). ABC de tecnovigilancia. https://paginaweb.invima.gov.co/images/pdf/tecnovigilancia/ABC\%20Tecnovigila ncia\%20INVIMA.pdf

Invima y Ministerio de Salud y Protección Social. (2013). ABC de dispositivos médicos. https://www.invima.gov.co/documents/20143/442916/abc dispositivosmedicos.pdf/d32f6922-0c50-bcaa-6b53-066edfb98274

Jezzini, A., Ayache, M., Elkhansa, L., Makki, B. y Zein, M. (2013). Effects of predictive maintenance $(\mathrm{PdM})$, proactive maintenace $(\mathrm{PoM})$ \& preventive maintenance $(\mathrm{PM})$ on minimizing the faults in medical instruments. En 2nd International Conference on Advances in Biomedical Engineering (pp. 53 - 56). IEEE Xplore.

López, F. (s. f.). Directrices para la dirección de proyectos.

https://www.aeipro.com/files/charlas\%20y\%20conferencias/2015/Badajoz\%2020 15/ISO_21500_seminario.pdf

Méndez, M., Londoño, L. y Camacho, N. (2013). Plan para la implementación del mantenimiento preventivo y/o correctivo en equipos WOS en campo CajuaPuerto Gaitán [Tesis de especialización]. Universidad Piloto de Colombia.

Ministerio de Salud y Protección Social. (2005, 27 de diciembre). Decreto 4725 de 2005. Por el cual se reglamenta el régimen de los registros sanitarios, permisos de comercialización y vigilancia sanitaria de los dispositivos médicos para uso humano. Diario Oficial 46134. https://docs.supersalud.gov.co/PortalWeb/Juridica/Decretos/D4725005.pdf 
Ministerio de Salud y Protección Social. (2007, 8 de noviembre). Resolución 4002 de 2007. Por el cual se adopta el Manual de Requisitos de Capacidad de Almacenamiento y/o Acondicionamiento para dispositivos médicos. Diario Oficial 46806.

https://docs.supersalud.gov.co/PortalWeb/Juridica/OtraNormativa/R_MPS_400 2 2007.pdf

Ministerio de Salud y Protección Social. (2008, 12 de diciembre). Resolución 4816 de 2008. Por el cual se reglamenta el Programa Nacional de Tecnovigilancia. Diario Oficial 47201.

http://www.saludcapital.gov.co/sitios/VigilanciaSaludPublica/Todo\%20IIH/Resol uci\%C3\%B3n\%204816\%20de\%202008.pdf

Ministerio de Salud y Protección Social. (2019). Sistema obligatorio de garantía de calidad en salud (SOGCS).

https://www.minsalud.gov.co/salud/PServicios/Paginas/sistema-obligatoriogarantia-calidad-SOGC.aspx

Ministerio de Salud y Protección Social (2019, 26 de noviembre). Resolución 3100 de 2019 Por la cual se definen los procedimientos y condiciones de inscripción de los prestadores de servicios de salud y de habilitación de servicios de salud y se adopta un manual de inscripción de prestadores y habilitación de servicios de salud. Diario Oficial 51149.

https://www.minsalud.gov.co/Normatividad Nuevo/Resoluci\%C3\%B3n\%20No.\% 203100\%20de\%202019.pdf

Morales, J., (2013). Apoyo en la gestión y desarrollo de las labores de mantenimiento a equipos biomédicos en la Clínica Fátima S.A en la ciudad de Pasto [Tesis de pregrado]. Universidad de Nariño.

Moreno, J., Angarita, I., Peñuela, M. y Ramírez, K. (2005). Aplicación del método delphi para determinar el costo máximo admisible en mantenimiento de los equipos médicos. Umbral Científico, 6, 41-44.

Naser H., Rami, O., Hamzeh, A. y Sammour, D. (2012). An intelligent healthcare management system: a new approach in work-order prioritization for medical equipment maintenance requests. Journal of Medical Systems, 36(2), 557-567.

Otzen, T. y Manterola, C. (2017). Técnicas de muestreo sobre una población en estudio. Int. J. Morphol, 35(1), 227-232. 
Primero, D., Díaz, J., García, L. y González, A. (2015). Manual para la gestión del mantenimiento correctivo de equipos biomédicos en la Fundación Valle de Lilí. Revista Ingeniería Biomédica, 9(18), 81-87.

Project Management Institute. (2017). Guía de los fundamentos para la dirección de proyectos. PMI Inc.

Ríos, A., Miguel, A., Rodríguez, L. y Chaparro, J. (2010). La ingeniería biomédica en Colombia: una perspectiva desde la formación del pregrado. Revista Ingeniería Biomédica, 4(7), 23-34.

Rocha, L., Rotger, V., Olviera, J. y Monzon, J. (2003). Clinical engineering methodology applied in the Tucumin's Public Health System: first results for $X$-ray service. Proceedings of the 25 Annual International Conference of the IEEE EMBS, Cancún, México.

Ruiz, C., Franco, A., Mejía, H. y Fajardo, R. (2010). Perspectivas en seguridad del paciente. Entrevista con líderes colombianos. Revista de Ingeniería Biomédica, $4(8), 10-21$.

Seiti, H. y Hafezalkotob, A. (2019). Developing the R.TOPSIS methodology for risk-based preventive maintenance planning: A case study in rolling Mil Company. Computer and Industrial Engineering, 128, 622-636.

Velazco, I. (s. f.). Normas legales a partir de la Constitución Política Colombiana para dispositivos médicos. Secretaría Distrital de Salud.

http://www.saludcapital.gov.co/sitios/VigilanciaSaludPublica/Todo\%20IIH/Norm atividad\%20en\%20Dispositivos\%20Medicos\%20[Modo\%20de\%20compatibilidad] . $\mathrm{pdf}$

Verastegui, J. (2014, 28 de mayo). ISO 2150o. Directrices para la dirección y gestión de proyectos. IV Congreso Internacional de Dirección de Proyectos, Guayaquil, Ecuador. 